\title{
Inside the large globule CB205: YSOs feeding multiple outflows
}

\author{
C. Codella ${ }^{1}$, J. Brand ${ }^{2}$, F. Massi ${ }^{3}$, J. G. A. Wouterloot ${ }^{4}$, and G. R. Davis ${ }^{4}$ \\ 1 INAF - Istituto di Radioastronomia, Sezione di Firenze, Largo E. Fermi 5, 50125 Firenze, Italy \\ e-mail: codella@arcetri.astro.it \\ 2 INAF - Istituto di Radioastronomia, via Gobetti 101, 40129 Bologna, Italy \\ 3 INAF - Osservatorio Astrofisico di Arcetri, Largo E. Fermi 5, 50125 Firenze, Italy \\ 4 Joint Astronomy Centre, 660 N. A'ohoku Place, University Park, Hilo, HI 96720, USA \\ Received 13 April 2006 / Accepted 27 June 2006
}

ABSTRACT

\begin{abstract}
Context. The molecular and continuum surveys at NIR and (sub-)mmwavelengths recently performed in the large and distant ( $\geq 1 \mathrm{kpc})$ Bok globules CB3 and CB34 are the framework of the present paper. With this multi-wavelength approach, it is possible to trace the hot jets and cold outflows driven by the (proto-)stars, to investigate how they interact with the surrounding medium, and to assess that in these globules star formation is a continuous process and not a unique event.

Aims. With the present work we continue our survey of a sample of large globules by investigating CB205. The aim is to carefully map the outflow motions and to locate the driving sources. The occurrence of outflows is used to identify the earliest star-forming regions.

Methods. Our analysis has been performed through JCMT observations at (sub)millimeter wavelengths of the continuum (850 and $450 \mu \mathrm{m})$ and $\mathrm{CO}(2-1)$ and (3-2) line emission.

Results. The continuum maps reveal three Class 0 candidates located in the western region of the globule, without NIR counterparts. The line maps show a complex scenario for the high velocity components with different clumps at different velocities. The outflow activity is concentrated around the Class 0 candidates. On the other hand, the NIR objects are associated with lower velocity outflow clumps.

Conclusions. The present results confirm that for CB205, star formation is propagating through the globule. The western part hosts the latest formation episode with Class 0 candidates feeding multiple outflows. We suggest that the eastern region, which is associated with a NIR cluster, reflects a later evolutionary phase, when the outflow is fading.
\end{abstract}

Key words. stars: formation - radio lines: ISM - ISM: jets and outflows - ISM: molecules - ISM: individual objects: CB205

\section{Introduction}

It is well-known that Bok globules (Bok \& Reilly 1947) are isolated dark molecular clouds closely associated with star formation (e.g., Clemens \& Barvainis 1988; Bourke et al. 1995; Henning \& Launhardt 1998, and references therein). Given their simple structure, Bok globules have often been used to investigate the process of isolated single star formation. The large majority of such molecular clouds host low-mass star formation, as confirmed by the low luminosities estimated for the embedded Young Stellar Objects (YSOs) in the 1-10 $L_{\odot}$ range (e.g., Yun \& Clemens 1990; Huard et al. 1999). A limited number (9, 15\%, according to Table 1 of Launhardt \& Henning 1997) of large globules stand out for their relatively large distances $(\sim 1 \mathrm{kpc})$, their large sizes $(\geq 1 \mathrm{pc})$ and high masses $\left(>100 M_{\odot}\right)$, and their association with intermediate-mass $\left(\geq 10 M_{\odot}\right)$ YSOs (Huard et al. 2000). To understand the nature of the star-forming regions (SFRs) hosted by the large globules, Huard et al. (2000) observed three of them (CB3, CB34, and CB205) at 850 and $450 \mu \mathrm{m}$, detecting the youngest YSOs, Class 0 sources, which can be recognised by bright submillimeter continuum emission due to the large amount of surrounding dust. Huard et al. (2000) suggested that, since sub-mm emission does not trace the previously detected, more evolved, NIR objects, star formation within these globules appears to be a continuous process rather than a single event.
Another precious observational tool that allows one to pinpoint Class 0 protostars is the observation of molecular outflows. As soon as a star forms, it generates fast and collimated jets that interact with the surrounding medium and create bipolar molecular outflows when the protostar is still in the main accretion phase (e.g., Bachiller 1996). With this in mind, in order to investigate the youngest stars and compare the characteristics derived from dust and gas emission, we are performing a study of the molecular content of the three large globules observed in the continuum by Huard et al. (2000). As a first step, we performed a survey of the molecular outflows in CB3 and CB34 by observing multi-line emission at mm-wavelengths (Codella \& Bachiller 1999; Codella et al. 2002; Codella \& Scappini 2003). Our highangular resolution maps of the $\mathrm{CO}$ outflows, first detected by Yun \& Clemens $(1992,1994)$, show that they are located in the region where the millimeter and submillimeter sources are present, offset from the position of the older NIR objects.

Next, we investigated the CB205 globule, also known as L810, which lies at a distance of $2 \mathrm{kpc}$ (Launhardt \& Henning 1997). IRAS $19433+2743$ is embedded in CB205, surrounded by a NIR nebulosity (Yun et al. 1993). The nebulosity is elongated in the N-S direction and contains at least 10 stars (Massi et al. 2004). The sub-mm observations by Huard et al. (2000) show two sources: SMM1, bright and extended, which coincides with the NIR cluster, and SMM2, which is fainter and located $\sim 44^{\prime \prime}$ to the SW, thus tracing another site of star formation. 
The maps of Huard et al. (2000) clearly show a ring-like and clumpy structure that connects SMM1 and SMM2, and that suggests the possible presence of additional embedded objects without NIR counterparts. From CO observations with rather limited resolution $\left(\sim 45^{\prime \prime}-48^{\prime \prime}\right)$, Xie \& Goldsmith (1990) and Yun \& Clemens (1994) have detected a single molecular outflow centred on the NIR nebula and with overlapping lobes. Their maps show that the main axis of the outflow has the same orientation (N-S) as the NIR nebula, thus suggesting a close connection between the shape of the nebula, the presence of the outflow, and its driving source. However, Clemens et al. (1996) showed that the outflow lobes are offset by about $30^{\prime \prime}$ to the West with respect to the IRAS position, calling for further high angular resolution observations to clarify the outflow morphology. We have thus investigated CB205 through a survey in the CO $J=2-1$ and 3-2 emission lines. We also performed submillimeter continuum observations. The main aims of this work are: (i) to map the outflow at higher angular resolution and to identify the driving source(s), as was already done for the other two large globules CB3 and CB34, and (ii) to use the occurrence of outflows to identify the youngest YSOs and thus to verify whether CB205 is associated with different YSOs' generations.

\section{Observations}

\subsection{Continuum}

In September 2003, the $450 \mu \mathrm{m}$ and $850 \mu \mathrm{m}$ continuum emissions towards CB205 have been simultaneously observed with the Submillimeter Common-User Bolometer Array (SCUBA; Holland et al. 1999) mounted on the 15-m James Clerk Maxwell Telescope (JCMT) at Mauna Kea (Hawaii, USA). A standard 64-point jiggle map has been made, which covers an area 2!3 in diameter, sampling the region centred at the position of IRAS 19433+2743: $\alpha_{2000}=19^{\mathrm{h}} 45^{\mathrm{m}} 23.8, \delta_{2000}=+27^{\circ} 50^{\prime} 57^{\prime \prime} .9$. Calibration has been performed by using water vapour monitor data at $186 \mathrm{GHz}$, scaled to give the optical depth at $225 \mathrm{GHz}$, and average ratios of $\tau(450 \mu \mathrm{m})$ and $\tau(850 \mu \mathrm{m})$ to $\tau(225 \mathrm{GHz})$. The final transition from instrumental parameters to $\mathrm{Jy} \mathrm{beam}^{-1}$ has been derived from a map of Mars made with the same chopping angle $\left(120^{\prime \prime}\right)$. Note that at $850 \mu \mathrm{m}$ a few bad bolometers have been blanked during the data reduction: this affects the eastern part of the map, producing a sharp decrease in the intensity on one side of the main peak (see Fig. 1). To increase the sensitivity of the maps, we have coadded the present data with those provided by the JCMT archives and published by Huard et al. (2000). The final rms values are $\sim 120 \mathrm{mJy} \mathrm{beam}^{-1}$ at $450 \mu \mathrm{m}$ and $\sim 16 \mathrm{mJy} \mathrm{beam}^{-1}$ at $850 \mu \mathrm{m}$, while the HPBWs are $10^{\prime \prime}$ and $16^{\prime \prime}$, respectively. The pointing uncertainty, $\leq 2$, , has been estimated by observing nearby source K3-50.

\subsection{Lines}

Maps of the CO $J=2-1(230.538 \mathrm{GHz})$ and $J=3-2$ $(345.796 \mathrm{GHz})$ emission were made with the 15-m JCMT during two runs in April and September 2003. The maps are centred at the IRAS $19433+2743$ position, and the observations were performed in raster mode with a spacing of $10^{\prime \prime}$ for the CO $J=$ 2-1 line, whereas for the $J=3-2$ emission we used a sampling of $8^{\prime \prime}$ except for the central part of CB205, where a smaller value $\left(4^{\prime \prime}\right)$ has been used. The $\mathrm{CO}$-free reference position was at offset $\left(0^{\prime \prime},-600^{\prime \prime}\right)$. We used the heterodyne receivers A3 $(J=$ $\left.2-1, H P B W=21^{\prime \prime}\right)$ and B3 $\left(J=3-2, H P B W=14^{\prime \prime}\right)$. The backend was the Digital Autocorrelation Spectrometer, set up with

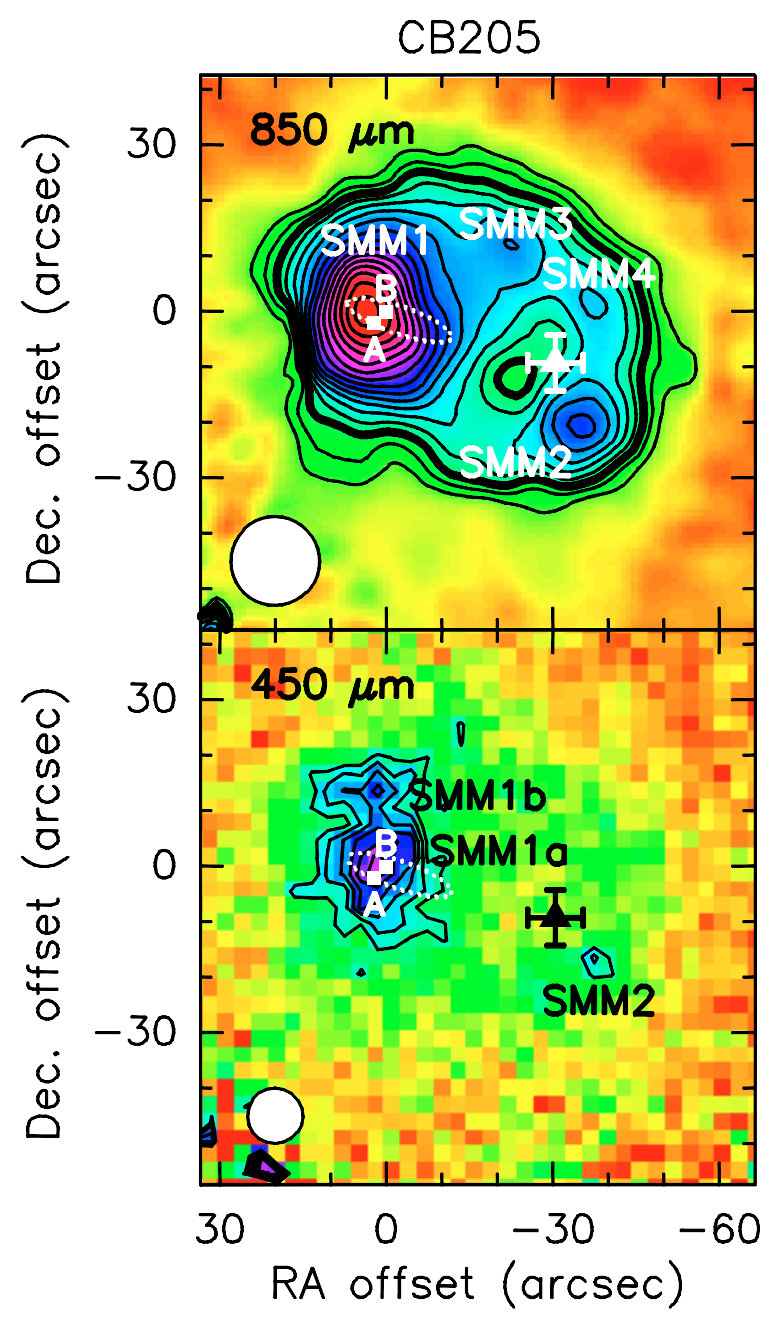

Fig. 1. $850 \mu \mathrm{m}$ (upper panel) and $450 \mu \mathrm{m}$ (lower panel) maps of CB205, obtained by coadding the present data with those from the JCMT archive and published by Huard et al. (2000). The filled squares point out the brightest NIR sources, A and B, as labeled by Massi et al. (2004). The origin is located at the position of IRAS 19433+2743, indicated by its uncertainty ellipse. The white circles show the JCMT beams (16" and $10^{\prime \prime}$ at 850 and $450 \mu \mathrm{m}$, respectively), while the filled triangle stands for the $\mathrm{H}_{2} \mathrm{O}$ maser detected by Neckel et al. (1985). The contour levels at $850 \mu \mathrm{m}$ range from $0.08(\sim 5 \sigma)$ to $0.32 \mathrm{Jy}$ beam $^{-1}$, whereas the contours at $450 \mu \mathrm{m}$ range from $0.38(\sim 3 \sigma)$ to $1.00 \mathrm{Jy} \mathrm{beam}^{-1}$. The steps correspond to about $1 \sigma$. Note that the $7 \sigma$ contour of the $850 \mu \mathrm{m}$ map has been drawn with a thicker line to stress the ring-like structure with a minimum at the centre.

a bandwidth of $500 \mathrm{MHz}$ and a consequent spectral resolution of 0.41 and $0.27 \mathrm{~km} \mathrm{~s}^{-1}$ at 231 and $346 \mathrm{GHz}$, respectively. The integration time (ON-source) per point for the $J=2-1$ and $3-2$ observations was respectively $120 \mathrm{~s}$ and $40 \mathrm{~s}$. The system temperature during the observations was about $280 \mathrm{~K}$ for the A3 receiver and about $690 \mathrm{~K}$ for the $\mathrm{B} 3$ receiver. Pointing was checked by observing W75N and NGC 7538 IRS1, and found to be accurate to within $\sim 3^{\prime \prime}$. The main beam efficiencies adopted to convert the antenna temperature into brightness temperature are 0.69 (at $230 \mathrm{GHz}$ ) and 0.63 (at $346 \mathrm{GHz}$ ); the average sensitivities $(1 \sigma)$ are about 80 and $200 \mathrm{mK}$, respectively.

\section{The cluster of YSOs}

The possibility that CB205 is hosting an aggregate of YSOs can be verified using Fig. 1, which shows the dust continuum maps 
Table 1. List of coordinates and peak fluxes of the sub-mm objects detected in CB205.

\begin{tabular}{lccccc}
\hline \hline SMM & $\begin{array}{c}\alpha_{2000} \\
(\mathrm{~h} \mathrm{~m} \mathrm{~s})\end{array}$ & $\begin{array}{c}\delta_{2000} \\
\left({ }^{\circ}{ }^{\prime \prime}\right)\end{array}$ & $\begin{array}{c}F_{\text {peak }}\left(\mathrm{Jy} \mathrm{beam}^{-1}\right) \\
(850 \mu \mathrm{m})\end{array}$ & $\begin{array}{c}M^{\dagger} \\
(450 \mu \mathrm{m})\end{array}$ & $M_{\odot}$ \\
\hline $1 \ddagger$ & 194524.3 & +275059.3 & 0.33 & - & 11 \\
$1 \mathrm{a} \ddagger$ & 194524.1 & +275058.8 & - & 1.10 & 8 \\
$1 \mathrm{~b} \ddagger$ & 194524.1 & +275112.8 & - & 0.79 & 3 \\
2 & 194521.2 & +275038.5 & 0.19 & 0.57 & 5 \\
3 & 194522.3 & +275110.6 & 0.17 & - & 3 \\
4 & 194521.1 & +275100.1 & 0.16 & - & 3 \\
\hline
\end{tabular}

$\dagger$ Total (gas+dust) mass, estimated from the $850 \mu \mathrm{m}$ observations, assuming $T_{\text {dust }}=25 \mathrm{~K}, \beta=1.3, \kappa_{230 \mathrm{GHz}}=0.005$, and a gas-to-dust ratio of 100 (see text); $\ddagger$ the source called SMM1 in the $850 \mu \mathrm{m}$ map is resolved in two objects, SMM1a and SMM1b, by the higher angular resolution observations at $450 \mu \mathrm{m}$. The SMM1a and SMM1b masses have been estimated by using the $450 \mu \mathrm{m}$ observations.

at 850 and $450 \mu \mathrm{m}$ (present plus Huard et al. 2000 data, see Sect. 2.1). The observed dust distributions of Fig. 1 obviously reproduce the images by Huard et al. (2000) showing a ringlike structure, but in addition the higher sensitivity allows us to identify two new unresolved sources detected with $S / N \sim 10$. These are labeled SMM3 and 4 in Fig. 1. The brightest source, SMM1, previously detected by Huard et al. (2000), is extended and associated with the brightest NIR objects, called A and B by Massi et al. (2004). The higher angular resolution of the $450 \mu \mathrm{m}$ observations shows that the extended structure of SMM1 is due to its association with at least two objects: SMM1a, centred on the brightest NIR counterparts, and SMM1b, which is definitely fainter and located $\sim 15^{\prime \prime}$ in the northern direction. At 850 and $450 \mu \mathrm{m}$, we also confirm the occurrence of the fainter SMM2 source, located 44" SW from SMM1. The coordinates and the peak flux densities of the four submillimeter sources are listed in Table 1. SMM2 and SMM4 have no NIR or optical counterparts, thus they are likely Class 0 objects. The nature of SMM3 is more uncertain since along its line-of-sight an optical star (\#16; Neckel \& Staude 1990) is visible, but it has no NIR counterpart. Further high-angular resolution observations at submillimeter wavelengths are needed in order to unambiguosly classify SMM3.

An estimate of the total mass of the objects has been derived from the $850 \mu \mathrm{m}$ total flux using the standard method (e.g., Huard et al. 1999), and assuming a dust opacity $\kappa_{v}=$ $\kappa_{230 \mathrm{GHz}}[v(\mathrm{GHz}) / 230]^{\beta}$ and $\kappa_{230 \mathrm{GHz}}=0.005 \mathrm{~cm}^{2} \mathrm{~g}^{-1}$ (Kramer et al. 1998), implying a gas-to-dust ratio of 100, a dust emissivity index $\beta=1.3$, and a dust temperature $T_{\text {dust }}=25 \mathrm{~K}$ (Huard et al. 2000). Since SMM2, SMM3, and SMM4 are not resolved, the total flux has been estimated by using the flux in the beam at these positions. The masses derived for $\mathrm{SMM} 1=\mathrm{SMM} 1 \mathrm{a}+\mathrm{SMM} 1 \mathrm{~b}, 11 M_{\odot}$, and $\mathrm{SMM} 2,5 M_{\odot}$, are in agreement with those given by Huard et al. (2000). The masses derived for SMM3 and SMM4, although they have to be taken with caution, are $\sim 3 M_{\odot}$. We used the $450 \mu \mathrm{m}$ total flux integrated over the HPBW to give a rough estimate of the SMM1a $\left(\sim 8 M_{\odot}\right)$ and SMM1b $\left(\sim 3 M_{\odot}\right)$ masses. The main uncertainties in mass estimates are due to the assumption of dust temperature: if we assume $T_{\text {dust }}$ in the $20-30 \mathrm{~K}$ range the total mass can vary up to a factor 1.4.

In conclusion, the present continuum observations confirm that SMM2, already classified by Huard et al. (2000) as Class 0, does not represent an isolated case and that the western part of the globule, away from the NIR cluster, hosts a very young generation of star formation, probably represented by three Class 0 candidates. That star formation occurs in the western region of $\mathrm{CB} 205$ and is also supported by the detection of an $\mathrm{H}_{2} \mathrm{O}$ maser between SMM1 and SMM2 as well as by the occurrence of a secondary peak appearing in $\mathrm{NH}_{3}(1,1)$ and $\mathrm{CS}(2-1)$ maps, tracing high-density $\left(\geq 10^{4} \mathrm{~cm}^{-3}\right)$ material (Neckel et al. 1985; Clemens et al. 1996). In view of this, SMM2, SMM3, and SMM4 are good candidates for the driving sources of the outflow motions in CB205, which we shall discuss next.

\section{Molecular gas kinematics: the multiple outflows}

In Figs. 2 and 3 we present the channel maps of the $\operatorname{CO}(2-1)$ and (3-2) emissions, respectively. The ambient LSR velocity is $+15.7 \mathrm{~km} \mathrm{~s}^{-1}$, from the $\mathrm{NH}_{3}(1,1)$ spectra reported by Lemme et al. (1996). At ambient velocities, we observe an apparent anticorrelation between the $\mathrm{CO}$ emission and the dust continuum maps. This could be an optical depth effect: $\mathrm{CO}$ emission is able to trace just the external part of the molecular cloud, missing the high-density interior, and consequently it cannot reproduce the $850 \mu \mathrm{m}$ map. On the other hand, a quite complex scenario emerges for the high-velocity gas, with several clumps at different velocities. The $\mathrm{CO}(2-1)$ maps indicate the presence of two blue-shifted clumps (hereafter called B1 and B2) and three red-shifted clumps (R1, R2, and R3). In addition, the higher angular resolution $\mathrm{CO}(3-2)$ maps, obtained with a very close spacing $(\sim H P B W / 3)$, allow us to identify a third blue-shifted clump, called B3. The positions of the clumps are: $\mathrm{B} 1\left(-10^{\prime \prime},-20^{\prime \prime}\right), \mathrm{B} 2$ and $\mathrm{R} 2\left(-20^{\prime \prime},+20^{\prime \prime}\right), \mathrm{B} 3\left(0^{\prime \prime}, 0^{\prime \prime}\right)$, $\mathrm{R} 1\left(-20^{\prime \prime},-20^{\prime \prime}\right)$, and R3 $\left(+20^{\prime \prime},-20^{\prime \prime}\right)$. The clumps B1 and R2 are the only clumps that have been resolved, with a beamdeconvolved size of $\sim 20^{\prime \prime}(\sim 0.25 \mathrm{pc}$ at $2000 \mathrm{pc})$. Thus, the single bipolar outflow with large red and blue lobes detected by Xie \& Goldsmith (1990) and Yun \& Clemens (1994) is resolved out into a number of separate outflow lobes here, suggesting a multiple outflow activity.

Figure 4 shows the superposition of the $\mathrm{CO}(3-2)$ channel maps at +9.7 and $+17.7 \mathrm{~km} \mathrm{~s}^{-1}$ giving a summary of the distribution of the red- (dotted line) and blue-shifted (continuous line) clumps. For the sake of clarity and to bring out the peak positions of B1 and B2, the contours of the blue emission are drawn with smaller steps. Given the high degree of confusion, it is quite risky to derive outflow geometries and to identify the driving sources. In fact, the observed outflow lobes could be the result of several overlapping outflows in the western part of the mm-continuum core of CB205 and, as a consequence, the driving sources should be looked for among SMM2, SMM3, and SMM4. However, focusing the attention on the clumps with the highest velocity detected in the $\mathrm{CO}(2-1)$ spectra, observed with a higher sensitivity, one may note that (i) B2 and R2 are spatially coincident, and (ii) R1 and B1 are nearly overlapping. One possible interpretation is that of a single outflow extending $\sim 80^{\prime \prime}(\sim 0.8 \mathrm{pc}$ at a distance of $2 \mathrm{kpc}$ ) along the NS direction. If the outflow is not highly collimated, and if the flow axis has a small inclination to the plane of the sky, then the propagating bow shocks can cause both red-and blue-shifted emission on the same side of the driving source, thus explaining the overlap of red and blue lobes seen in Fig. 4. The best candidate for the driving source in this scenario would be SMM3. Alternatively, the four lobes with the highest velocities might be tracing two outflows with their flow axes located roughly along the line-of-sight; in this case SMM3 could contain the driving YSOs of the northern outflow (R2, B2), while SMM2 would be the best candidate for the YSO associated with the southern one $(\mathrm{R} 1, \mathrm{~B} 1)$. Interferometry, 
CB205 CO(2-1)

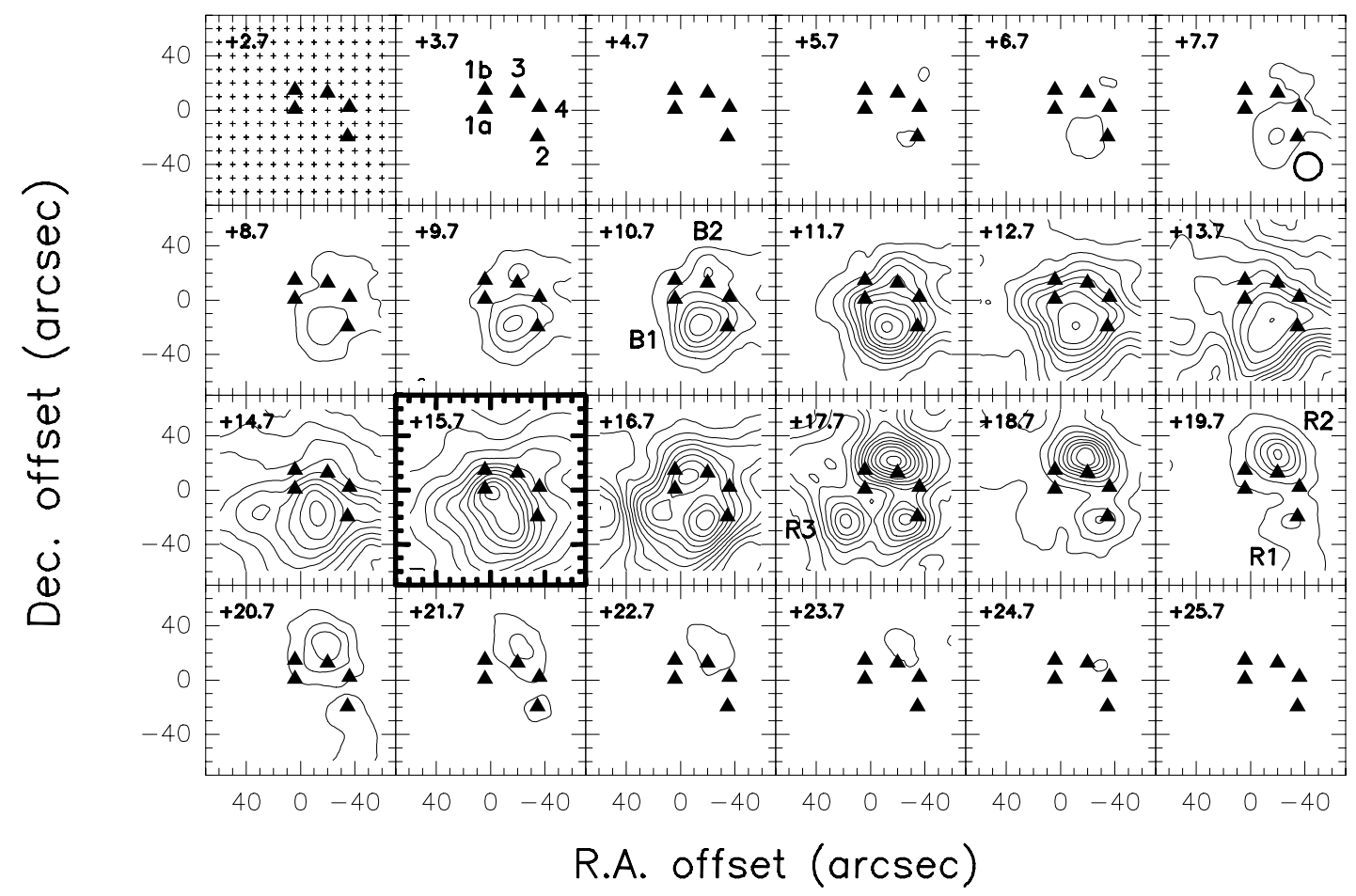

Fig. 2. Channel maps of the CO $J=2-1$ emission towards CB205. Each panel shows the emission integrated over a velocity interval of $1 \mathrm{~km} \mathrm{~s}^{-1}$ centred at the value given in the left corner. The thick box indicates the ambient velocity range $\left(+15.7 \mathrm{~km} \mathrm{~s}^{-1} ; 1996\right)$. The origin is located at the IRAS $19433+2743$ position. The five filled triangles indicate the locations of the $850 \mu \mathrm{m}$ continuum sources, the empty circle in the top right panel shows the JCMT beam $(H P B W)$, while the small crosses mark the observed positions. The labels B1, B2, R1, R2, and R3 identify lobes of the different molecular outflows. The contours range from $0.35 \mathrm{~K} \mathrm{~km} \mathrm{~s}^{-1}\left(\sim 5 \sigma\right.$, where $\sigma$ is the rms noise in the map) to $10.85 \mathrm{~K} \mathrm{~km} \mathrm{~s}^{-1}$ in steps of $0.70 \mathrm{~K} \mathrm{~km} \mathrm{~s}^{-1}$.

CB205 CO(3-2)

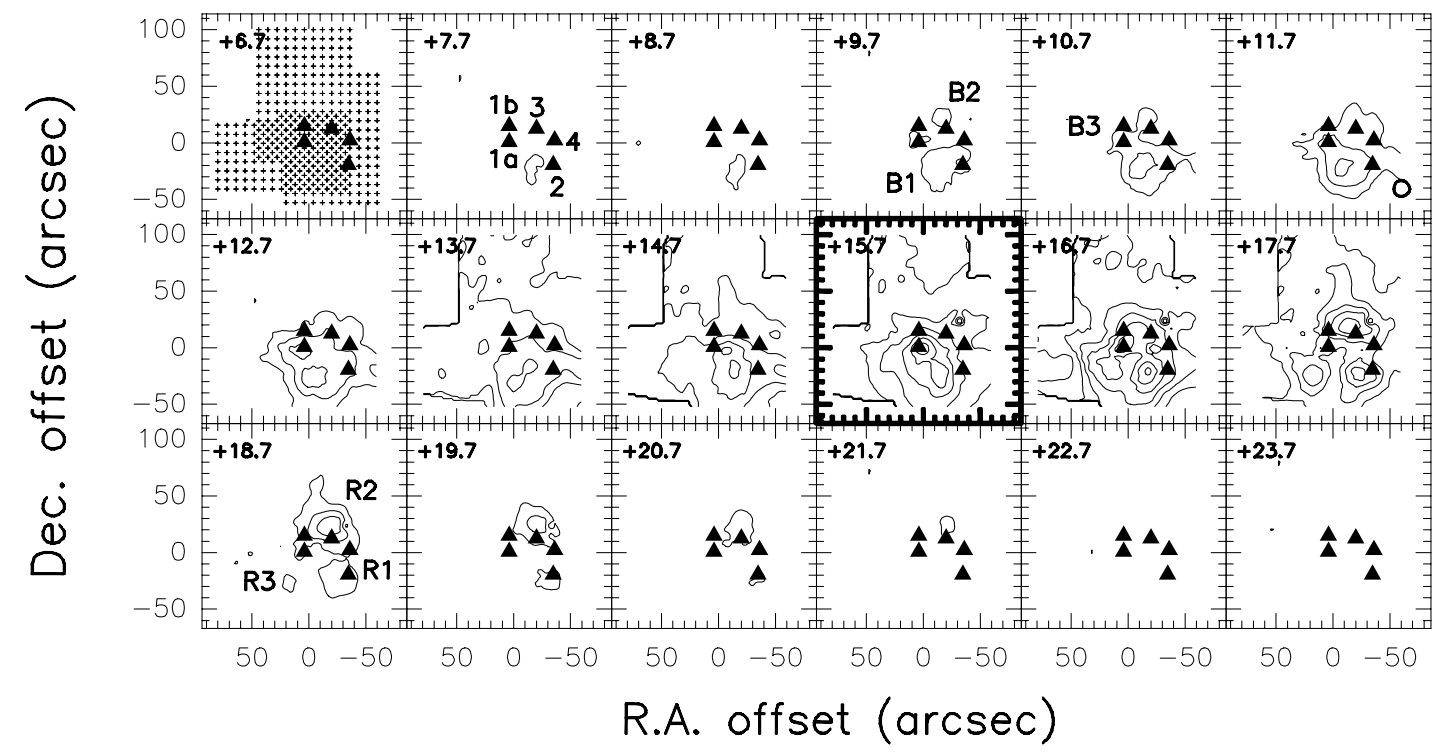

Fig. 3. Channel maps of the CO $J=3-2$ emission towards CB205. Each panel shows the emission integrated over a velocity interval of $1 \mathrm{~km} \mathrm{~s}^{-1}$ centred at the value given in the left corner. Symbols are drawn as in Fig. 2. The contours range from $0.75 \mathrm{~K} \mathrm{~km} \mathrm{~s}^{-1}(\sim 5 \sigma$, where $\sigma$ is the rms noise in the map) to $14.25 \mathrm{~K} \mathrm{~km} \mathrm{~s}^{-1}$ in steps of $1.50 \mathrm{~K} \mathrm{~km} \mathrm{~s}^{-1}$.

or even single-dish observations of emission from higher excitation $\mathrm{CO}$ transitions would be instructive to resolve possible jet-like structures and thus help to unravel this situation.
On the eastern side of the dust continuum core, as clearly shown by the higher-sensitivity $\mathrm{CO}(2-1)$ data, SMM1a and SMM1b are associated with lower-velocity emission, and 


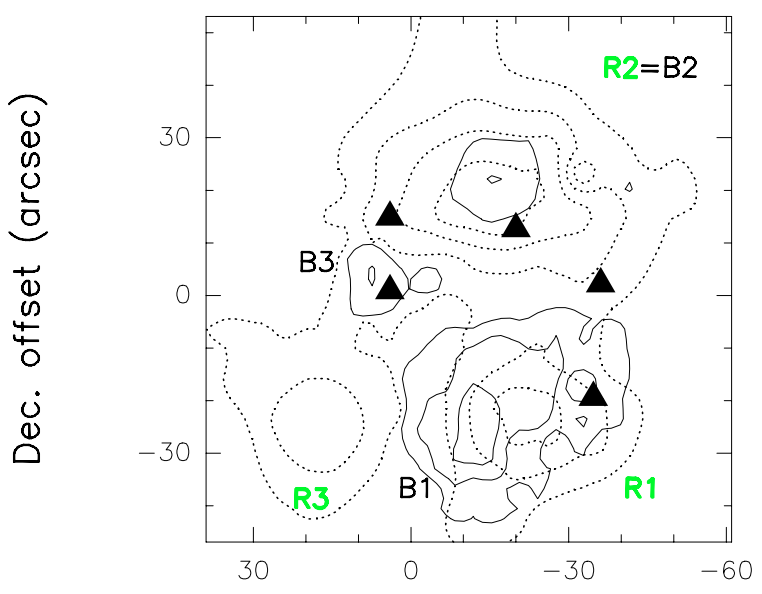

R.A. offset (arcsec)

Fig. 4. Summary of the distributions of the blue- (continuous lines and black labels) and red-shifted (dotted lines and gray labels) clumps. The map reports the zoom of the $\mathrm{CO}(3-2)$ channel maps at +9.7 and $+17.7 \mathrm{~km} \mathrm{~s}^{-1}$ (see Fig. 3). To point out the peak positions of B1 and B2, the blue emission is drawn starting from the $5 \sigma$ level and successively every $3 \sigma$. The filled triangles stand for the submillimeter objects (see Fig. 1).

in particular with the clumps B3 and R3. The lack of high velocity emission at the SMM1a,b positions could be due to geometrical effects, or it could reflect a different, later evolutionary phase of the driving YSO, when the outflow is fading.

In conclusion, we suggest that the western region of the globule is hosting the main outflow activity and thus the actual star-forming process. The observed high-velocity outflowing gas cannot be the cause of the extended NIR nebulosity, located along the N-S direction and centred on the IRAS point source position. Thus the NIR nebula could eventually be connected with a later phase of outflow activity, as was possibly also indicated by the relatively lower velocity clumps.

Finally, Fig. 5 shows the $\mathrm{CO}(2-1)$ and (3-2) spectra observed towards the outflow clumps. The profiles are skewed to the red with respect to the ambient velocity (dashed lines), measured through $\mathrm{NH}_{3}$ emission by Lemme et al. (1996). This is likely to be self-absorption, due to the high opacity expected for the $\mathrm{CO}$ emission in combination with a temperature gradient in the core. If we assume that the temperature increases in the central part of the region hosting the YSOs, this suggests that the outer layers of the cloud, as traced by $\mathrm{CO}$, are expanding.

Rotation or the presence of different clumps at different velocities are unlikely explanations of the profile shapes: the CO channel maps do not show any dynamical signature of the former, while the spectra outside the mm-continuum core, at RA and/or Dec offsets $\geq 40^{\prime \prime}$, show symmetric profiles, centred on the systemic velocity. In addition, Xie \& Goldsmith (1990) show that the ${ }^{12} \mathrm{CO}(1-0)$ spectrum taken at the $\left(0^{\prime \prime}, 0^{\prime \prime}\right)$ position peaks slightly red-wards with respect to the ${ }^{13} \mathrm{CO}(1-0)$ line, supporting the opacity effect.

By comparing the spectra of two rotational CO lines, $J=2-1$ and 3-2 in this case, it is possible to derive an excitation temperature (e.g., Bachiller \& Tafalla 1999) and, since CO is expected to be thermalised, obtain kinetic temperatures. Given the complex geometry associated with the multiple outflows, two representative positions in the blue and the red lobes have been considered. Following the $\mathrm{CO}$ channel maps, we have chosen the brightest clumps, i.e., B1 and R2. To allow the spectra to be compared, the different angular resolutions of the two transitions have been taken into account, convolving the $J=3-2$ emission to the HPBW of the 2-1 (21"). The convolved $\mathrm{CO}(3-2)$ emission is weaker by $\sim 10 \%$, indicating dilution and confirming that the high-velocity CO clumps are smaller than the JCMT HPBW at the $\mathrm{CO}(2-1)$ frequency. Figure 6 (right panel) shows the behaviour of R32, defined as the ratio between the observed brightness temperatures of the $J=3-2$ and 2-1 transitions, as a function of the excitation temperature $\left(T_{\mathrm{ex}}\right)$ in the optically thick (continuous line) and optically thin (dashed line) regimes. If the $\mathrm{CO}$ emission is optically thin and $T_{\mathrm{ex}}$ is the same for both transitions, $\mathrm{R} 32=2.25 \mathrm{e}^{-16.6 / T_{\mathrm{ex}}}$; for optically thick $\mathrm{CO}$ emission, R32 = $1.5\left(\mathrm{e}^{11.1 / T_{\mathrm{ex}}}-1\right)\left(\mathrm{e}^{16.6 / T_{\mathrm{ex}}}-1\right)^{-1}$. In practice, for reasonable values of the temperature, in the thick case R32<1, while in the thin limit the curve tends monotonically to $\sim 2.25$ when $T_{\text {ex }}$ tends to infinity. The intermediate cases fall in between the two curves. The left panels of Fig. 6 show R32 (black line) as a function of velocity towards the red- (R2 clump; upper panel) and blue-shifted (B1 clump; lower panel) lobes. For reference the $\mathrm{CO}(3-2)$ profiles are also shown (not to scale; grey line). The ratio R32 at the ambient velocity, marked by the grey vertical line, assumes values of around $0.7-0.8$ that can be explained by optically thick emission at $T_{\text {ex }} \simeq 10-15 \mathrm{~K}$. On either side, R32 decreases monotonically with velocity to values of around 0.4 ; this could be due to a variation of the excitation temperature with velocity, or, more reasonably, it could reflect a thinner emission due to the smaller amount of gas outflowing at high velocities. If we assume the optically thin regime at the highest velocities, we derive temperatures around $10 \mathrm{~K}$. Note that the JCMT archives provide some ${ }^{13} \mathrm{CO}(2-1)$ spectra observed in 1990 around the R3 position. A comparison with the $\mathrm{CO}(2-1)$ profiles indicates that the $\mathrm{CO}$ emission at the systemic velocity is optically thick $\left(T_{\mathrm{MB}}\left({ }^{12} \mathrm{CO}\right) \sim 3 T_{\mathrm{MB}}\left({ }^{13} \mathrm{CO}\right) ; \tau_{12}>\right.$ 10). This supports the assumption of the optically thick regime at these velocities for the B1 and R2 clumps also. Unfortunately, the ${ }^{13} \mathrm{CO}(2-1)$ spectra do not allow us to derive an opacity estimate at high velocities.

To understand the physics of the outflow gas, we need to estimate mass, energy, and momentum, which in turn depend on optical depth and excitation temperature. Given the complexity of the CB205, we have chosen to sample the whole region associated with the outflow region. Moreover, we used the derived excitation temperature $(10 \mathrm{~K})$, and we conservatively assumed an optical depth $\tau$ in the range $0.1-1$. The standard procedure given, e.g., by Lada (1985) or Margulis \& Lada (1985) has been used. We integrated the emission in bins of $2 \mathrm{~km} \mathrm{~s}^{-1}$ at each position, taking the difference between the mean velocity of each bin and the systemic velocity as the outflow velocity. As we are only interested in the outflowing gas, the central part of the emission line was excluded. For each bin, we then calculated the $\mathrm{CO}$ column densities $\left(\propto \int T \mathrm{~d} v\right)$ and the mass, assuming a $\mathrm{CO}$ to $\mathrm{H}_{2}$ abundance ratio of $10^{-4}$. Likewise, we derive the momentum $P \propto \int T \mathrm{~d} v \times v$ and the energy $E \propto \int T \mathrm{~d} v \times v^{2}$ for each bin. The total mass, momentum, and energy of the outflow are then derived by summing over all bins and all positions. A dynamical timescale (age) for the outflow is defined as $t_{\text {dyn }}=R / V$, with $R$ the size of the flow and $V$ the characteristic outflow velocity. The outflow's mechanical luminosity is then $L_{\text {mech }}=E / t_{\text {dyn }}$, and the mass loss rate $\dot{M}=M / t_{\text {dyn }}$.

We find a total mass of 2-4 $M_{\odot}$ (for $\tau=0.1-1$ ), equally distributed between the red- and blue-shifted emission, whereas the momentum $P \simeq 8-12 M_{\odot} \mathrm{km} \mathrm{s}^{-1}$. An average dynamical 


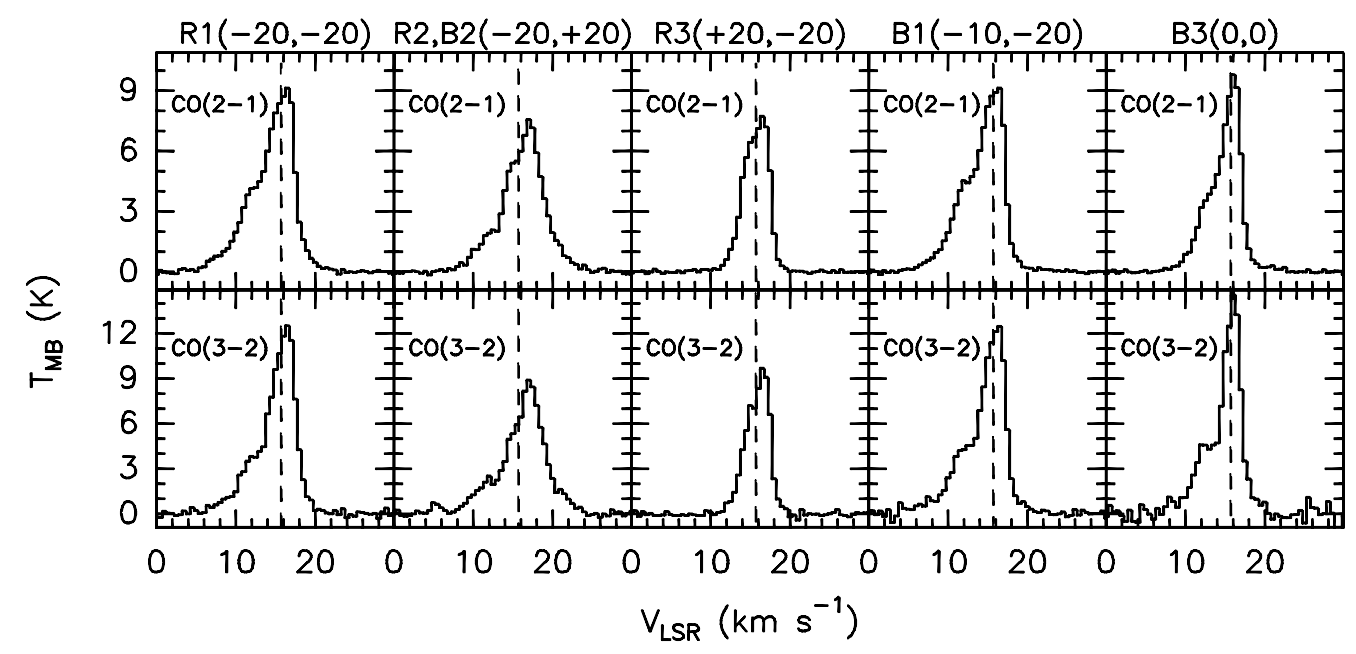

Fig. 5. Examples of the $\mathrm{CO}(2-1)$ and (3-2) profiles observed towards the high-velocity clumps located in CB205 and revealed by the channel maps (Fig. 2). Transition and angular offset are indicated. Note that B2 and R2 are positionally coincident and that the R2, B2 CO(3-2) spectrum suggests a blue-shifted peak at $\sim+5 \mathrm{~km} \mathrm{~s}^{-1}$ that is not bright enough to be mapped in Fig. 3. The dashed lines indicate the ambient LSR velocity, according to the $\mathrm{NH}_{3}(1,1)$ measurements by Lemme et al. (1996).

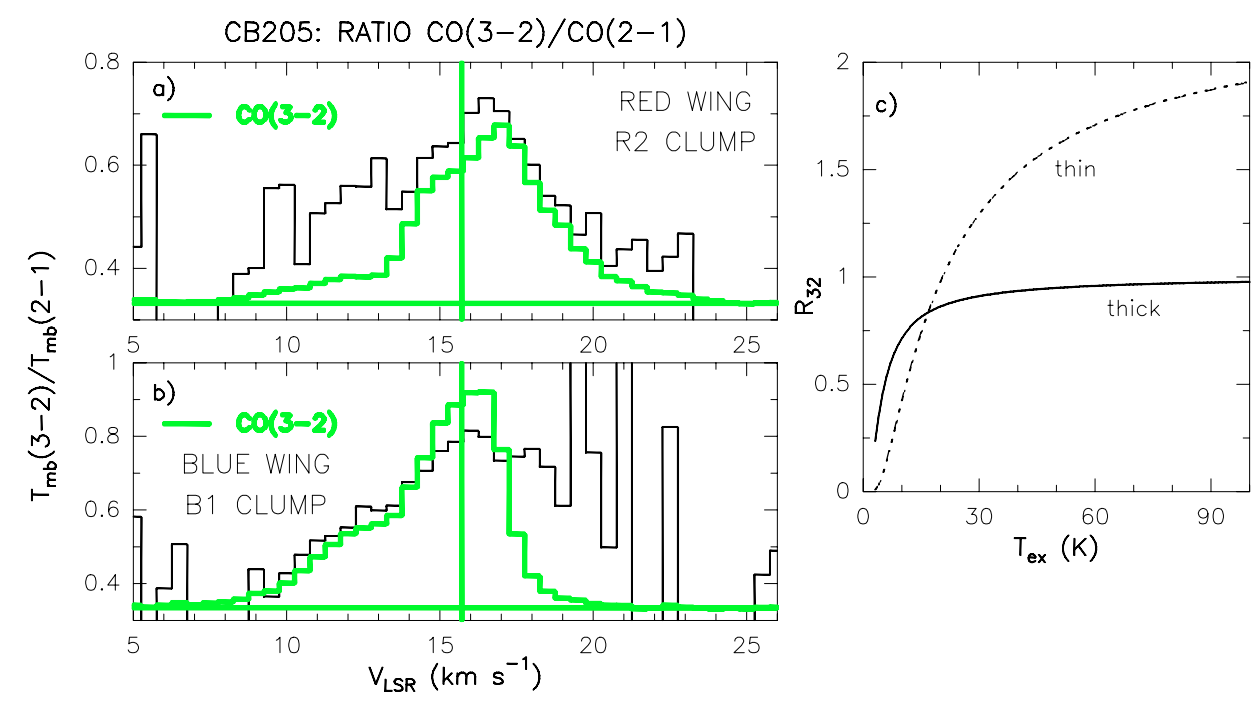

Fig. 6. The black line shows the intensity ratio $R_{32}$ ratio across the line profiles taken towards the red a) and blue-shifted b) brightest lobes of the CB205 outflows (see text). As a reference, the grey histogram shows the $\mathrm{CO}(3-2)$ profiles (not to scale). The vertical grey line indicates the ambient LSR velocity. $R_{32}$ decreases from a value of $\sim 0.7-0.8$ at the ambient velocity in the optically thick regime to values at around 0.4 at high velocities, where the emission is expected to be optically thinner. c) $R_{32}$ as a function of the excitation temperature in the optically thin (dot-dashed line) and thick (continuous line) regimes.

timescale of about $5 \times 10^{4} \mathrm{yr}$ has been deduced, from which it follows that $L_{\text {mech }} \sim 0.08-0.11 L_{\odot}$, and $\dot{M} \sim(6-8) \times$ $10^{-5} M_{\odot} \mathrm{yr}^{-1}$.

The parameters have been derived separately from the $J=$ 3-2 and 2-1 lines: the estimates are the same only if $\tau(3-2) \leq$ $\tau(2-1)$. This result gives us some indications about the density of the outflowing gas: by means of statistical-equilibrium calculations and assuming the derived $T_{\mathrm{ex}}=10 \mathrm{~K}$, we verified that the constraint on the optical depths is satisfied when the $\mathrm{H}_{2}$ density is in the range $10^{3}-10^{5} \mathrm{~cm}^{-3}$.

Finally, to estimate the uncertainties introduced by the lack of geometrical correction, we have assumed a quite extreme situation, where the flow axis is close to the plane of sky, with an inclination $i=20^{\circ}$, and then we have corrected the outflow velocity by $\sin i$. This leads to $P \simeq 23-34 M_{\odot} \mathrm{km} \mathrm{s}^{-1}, L_{\text {mech }} \simeq$ 1.9-2.6 $L_{\odot}$, and $\dot{M} \simeq(16-22) \times 10^{-5} M_{\odot} \mathrm{yr}^{-1}$, for $\tau=0.1-1.0$.
If we compare the derived outflow properties with those reported by the literature (e.g. Cabrit \& Bertout 1992; Shepherd \& Churchwell 1996) we can roughly estimate the bolometric luminosity $\left(L_{\mathrm{bol}}\right)$ of the driving YSO(s). In particular, the $L_{\mathrm{mech}}-L_{\mathrm{bol}}$ and the $\dot{M}-L_{\text {bol }}$ relationships indicate a driving YSO with $L_{\mathrm{bol}}$ in the range between $\sim 10 L_{\odot}$ (no geometrical correction) and $\sim 100 L_{\odot}\left(i=20^{\circ}\right)$ for the CB205 outflows. The estimates have to be considered upper limits, because we are observing multiple outflows and multiple YSOs.

Note that close to the SW edge of the globule, a very narrow $\left(1.3 \mathrm{~km} \mathrm{~s}^{-1}\right)$ spectral component at $+20.1 \mathrm{~km} \mathrm{~s}^{-1}$ appears. Considering its narrowness, it is plausible that rather than an outflowing component, this could simply be due to a quiescent cloud in the foreground or background of CB205. To derive an estimate of the contribution of this spectral component to the outflow parameters derived for the red-shifted emission, we have recalculated the energetics by excluding the spectra of 
the SW edge of CB205: the conclusion reported above about the YSOs luminosities remains the same, since mass, momentum, luminosity, and mass-loss rate are reduced by a factor between $10 \%$ and $20 \%$.

As a first step in investigating the interaction of the outflows with the ambient medium, we used the JCMT antenna to search for emission from $\mathrm{SiO}$, which is a standard tracer of shocked gas. We selected the high-excitation $\left(E_{\mathrm{u}}=31 \mathrm{~K}\right) J=5-4$ transition at $217.1 \mathrm{GHz}\left(H P B W=22^{\prime \prime}\right)$. The $\mathrm{SiO}$ emission has been searched for towards the brightest clumps, B1 and R2, without success and with an rms of $\sim 14 \mathrm{mK}$. Only through maps of all outflows in different $\mathrm{SiO}$ transitions will we be able to clarify if this lack of detection is due to an excitation effect or to a real low $\mathrm{SiO}$ abundance.

\section{Conclusions}

We have investigated the SFR associated with the large globule CB205 through continuum and molecular line observations at (sub-)millimeter wavelengths. The main findings are:

1. The 850 and $450 \mu \mathrm{m}$ continuum maps confirm the images published by Huard et al. (2000) showing a clumpy ringlike structure. The brightest source, SMM1, associated with the NIR cluster, has been resolved in two objects, SMM1a and SMM1b. A fainter source, SMM2, is located at 44" SW from SMM1, tracing another site of star formation. In addition, the present maps show the ring-like filament to be resolved into two additional, faint sources: SMM3 and SMM4. CB205 is thus associated with a collection of YSOs: the western region of the globule, without NIR counterparts, hosts the more recent phases of star formation, with SMM2, SMM3, and SMM4 being Class 0 candidates.

2. The $\mathrm{CO}(2-1)$ and (3-2) maps show that the high-velocity structure of CB205 is very complex with different clumps at different velocities. The outflow activity is concentrated in the western region of the globules, around the fainter SMM2, SMM3, and SMM4 sources. These are the best candidates to be the driving sources of the highest velocity clumps, and thus to trace the most recent star formation episode in CB205, in agreement with the continuum results. On the other hand, SMM1a and SMM1b, which are the counterparts of the NIR cluster, are associated only with a relatively lowvelocity outflow, suggesting a later evolutionary phase of the driving YSO, when the outflow is fading.

Acknowledgements. We are grateful to R. Cesaroni for his critical reading of the manuscript. The JCMT is operated by the Joint Astronomy Centre on behalf of the Particle Physics and Astronomy Research Council of the United Kingdom, the Netherlands Organisation for Scientific Research, and the National Research Council of Canada. The data reported in the present paper have been obtained thanks to the M03BI06 and M03AD14 JCMT projects. JCMT archive data were obtained from the Canadian Astronomy Data Netherlands Organisation for Scientific Research Centre, which is operated by the Herzberg Institute of Astrophysics, National Research Council of Canada.

\section{References}

Bachiller, R. 1996, ARA\&A, 34, 111

Bachiller, R., \& Tafalla, M. 1999, Bipolar Molecular Outflows, in The Physics of Star Formation and Early Stellar Evolution, NATO Advanced Science Institute, ed. C. J. Lada (Kluwer: Dordrecht), 227

Bok, B., \& Reilly, E. F. 1947, ApJ, 105, 255

Bourke, T. L., Hyland, A. R., \& Robinson, G. 1995, MNRAS, 276, 1052

Cabrit, S., \& Bertout, C. 1992, A\&A, 261, 274

Clemens, D. P., \& Barvainis, R. 1988, ApJS, 68, 257

Clemens, D. P., Berkovitch, M., Yun, J. L., Patel, N., \& Xie, T. 1996, ApJ, 457, 743

Codella, C., \& Bachiller, R. 1999, A\&A, 350, 659

Codella, C., \& Scappini, F. 2003, MNRAS, 344, 1257

Codella, C., Scappini, F., Bachiller, R., \& Benedettini, M. 2002, MNRAS, 331, 893

Henning, Th., \& Launhardt, R. 1998, A\&A, 338, 223

Holland, W. S., Robson, E. I., Gear, W. K., et al. 1999, MNRAS, 303, 659

Huard, T. L., Sandell, G., \& Weintraub, D. A. 1999, ApJ, 526, 833

Huard, T. L., Weintraub, D. A., \& Sandell, G. 2000, A\&A, 362, 635

Kramer, C., Alves, J., Lada, C., et al. 1998, A\&A, 329, L33

Lada, C. J. 1985, ARA\&A, 23, 267

Launhardt, R., \& Henning, Th. 1997, A\&A, 326, 329

Lemme, C., Wilson, T. L., Tieftrunk, A. R., \& Henkel, C. 1996, A\&A, 312, 585

Margulis, M., \& Lada, C. J. 1985, ApJ, 299, 925

Massi, F., Codella, C., \& Brand, J. 2004, A\&A, 419, 241

Neckel, T., \& Staude, H. J. 1990, A\&A, 231, 165

Neckel, T., Chini, R., Güsten, R., \& Wink, J. E. 1985, A\&A, 153, 253

Shepherd, D. S., \& Churchwell, E. 1996, ApJ, 472, 225

Xie, T., \& Goldsmith, P. F. 1990, ApJ, 359, 378

Yun, J. L., \& Clemens, D. P. 1990, ApJ, 365, L73

Yun, J. L., \& Clemens, D. P. 1992, ApJ, 385, L21

Yun, J. L., \& Clemens, D. P. 1994, ApJS, 92, 145

Yun, J. L., Clemens, D. P., McCaughrean, M. J., \& Rieke, M. 1993, ApJ, 408, L101 